Supporting Information

\title{
Insights into the Assembly of the Pseudogemini Surfactant at the Oil/Water Interface: A Molecular Simulation Study
}

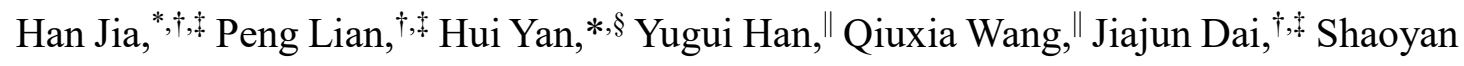
Wang, $^{\dagger,+}$ Zihao Tian ${ }^{\dagger+\star}$ and Daofan Wang ${ }^{\dagger \dagger}$

$\dagger$ Key Laboratory of Unconventional Oil \& Gas Development (China University of Petroleum (East China)), Ministry of Education, Qingdao, 266580, China.

† Shandong Key Laboratory of Oilfield Chemistry, School of Petroleum Engineering, China University of Petroleum (East China), Qingdao, 266580, China.

$\S$ School of Pharmacy, Liaocheng University, Liaocheng, 252059, China.

|| Bohai Oilfield Research Institute, Tianjin Branch, CNOOC China Limited, Tianjin, 300459, China.

*E-mail: jiahan@upc.edu.cn (H.J.).

*E-mail: yanhuilcu@163.com (H.Y.).

Number of pages: 10

Number of figures: 8

Number of schemes: 0

Number of tables: 1 

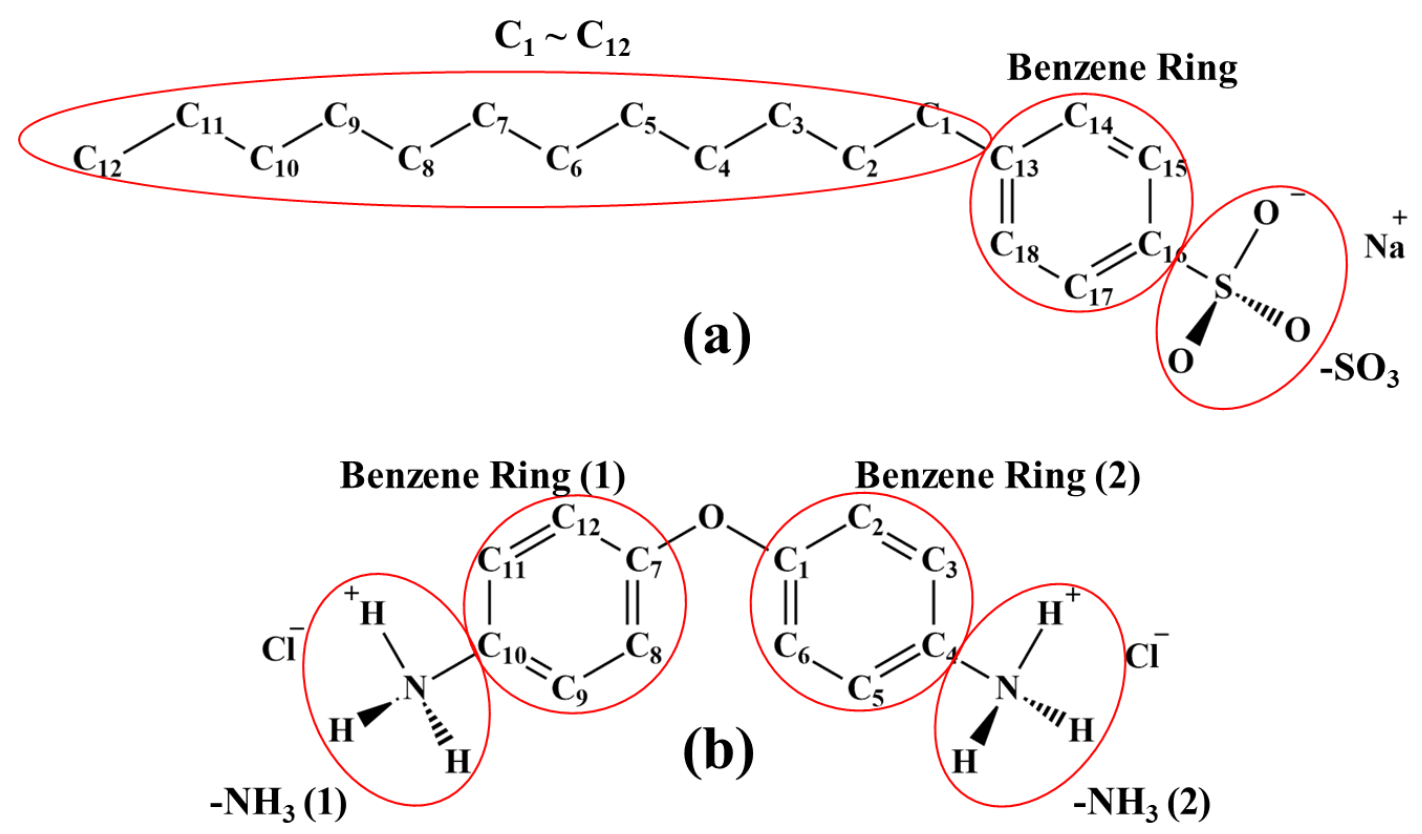

Figure S1. Molecular structures of (a) SDBS and (b) ODC. 
Table S1. Compositions of the solvent molecules

\begin{tabular}{ccccc}
\hline & \multicolumn{2}{c}{ Number of solvent molecules } & \\
\cline { 2 - 4 } System & Water & Toluene & Octane & Box Size \\
\hline SDBS & 36461 & 2940 & 1962 & $10.2 \times 10.2 \times 20.4 \mathrm{~nm}^{3}$ \\
ODC & 36783 & 2940 & 1962 & $10.2 \times 10.2 \times 20.4 \mathrm{~nm}^{3}$ \\
SDBS/ODC & 36361 & 2940 & 1962 & $10.2 \times 10.2 \times 20.4 \mathrm{~nm}^{3}$ \\
\hline
\end{tabular}




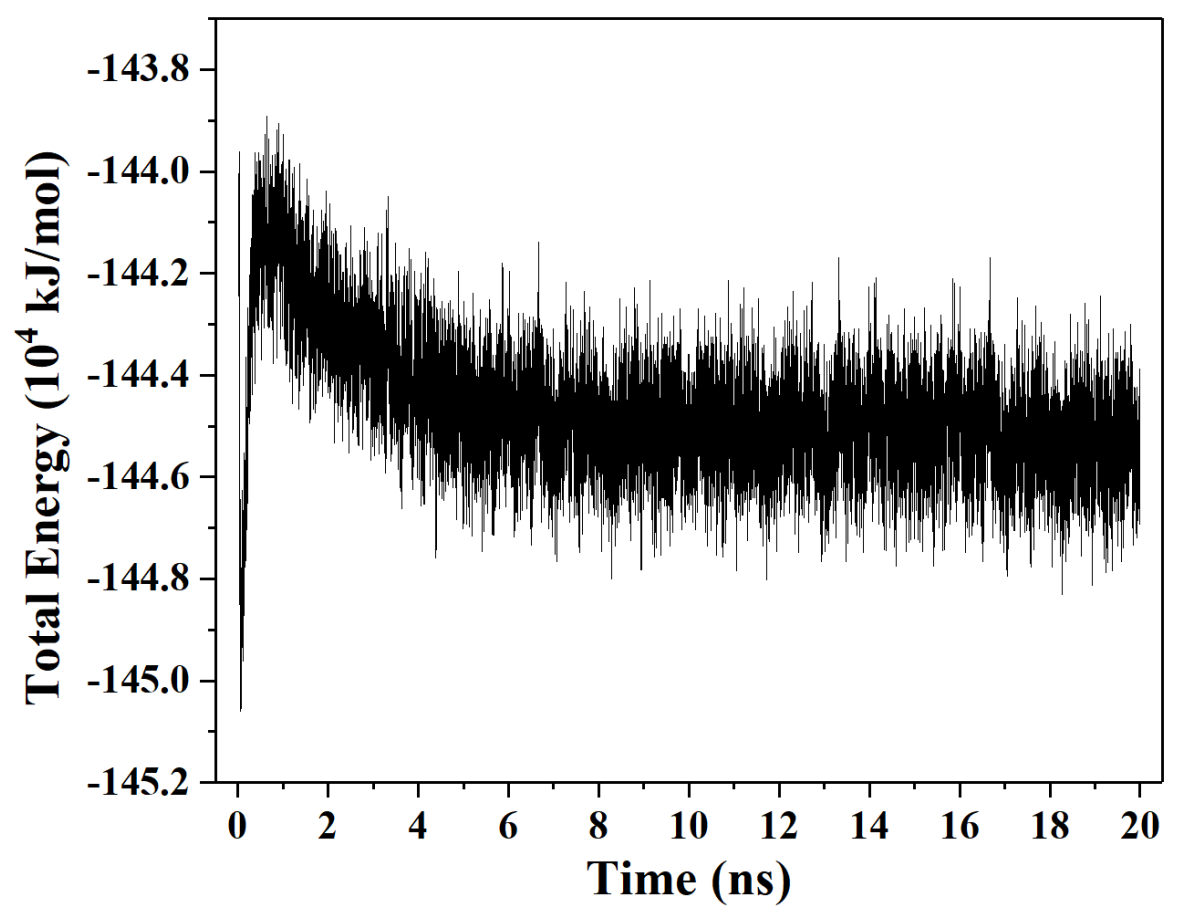

Figure S2. Total energy curve of the single SDBS system in the simulation process. 


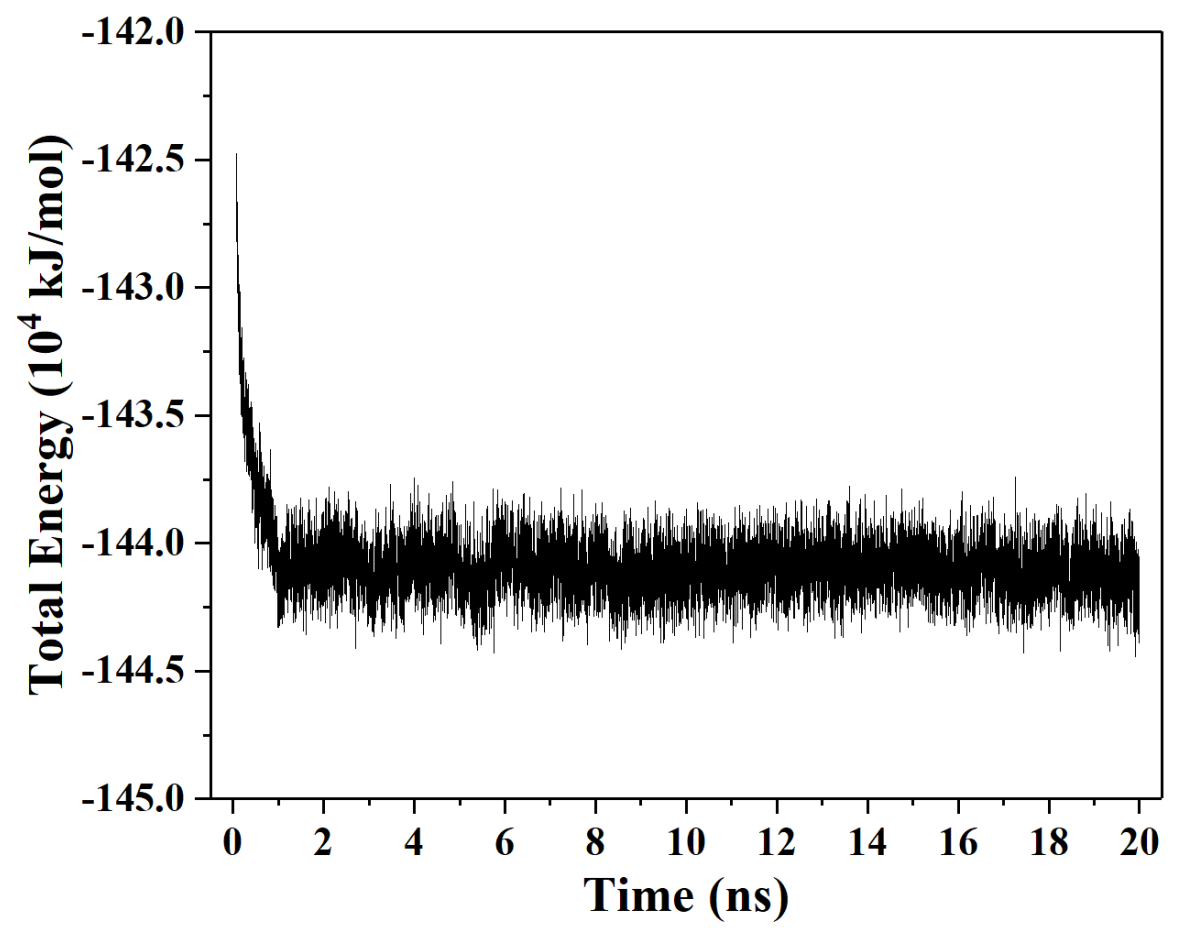

Figure S3. Total energy curve of the single ODC system in the simulation process. 


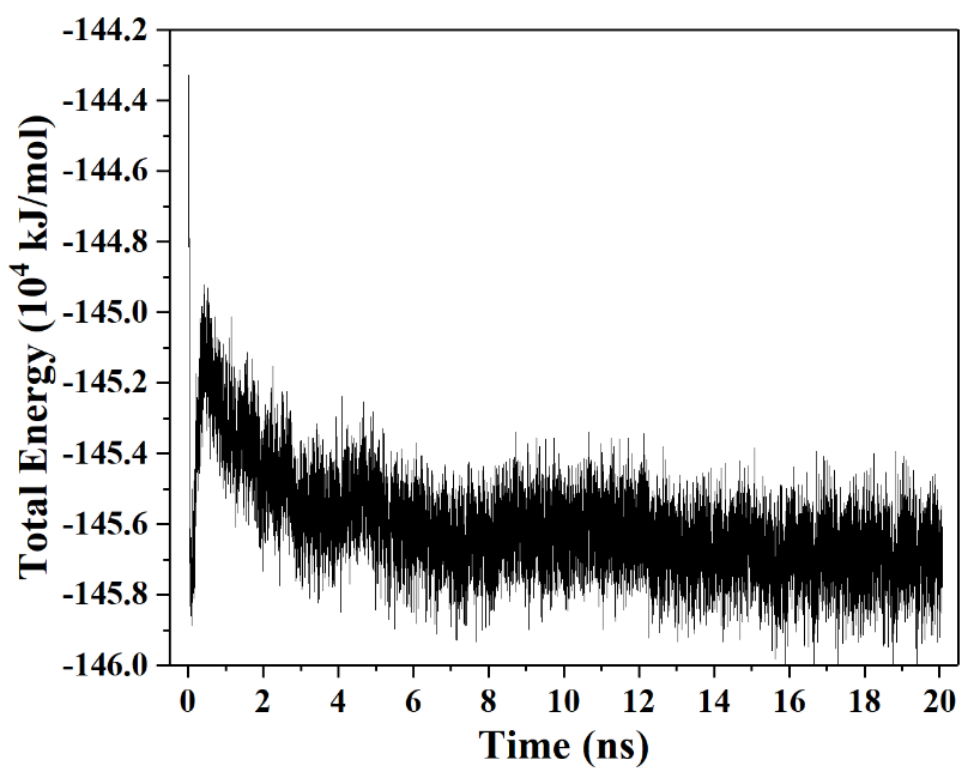

Figure S4. Total energy curve of the mixed SDBS/ODC system with the molecular ratio of $4: 1$ in the simulation process. 


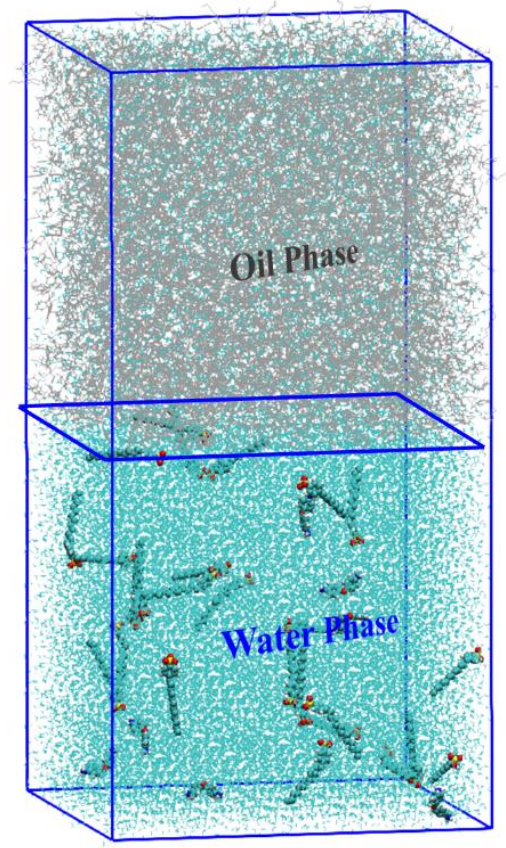

Figure S5. Snapshot at the beginning (0 ns) of the simulation process in the SDBS/ODC mixed system. 

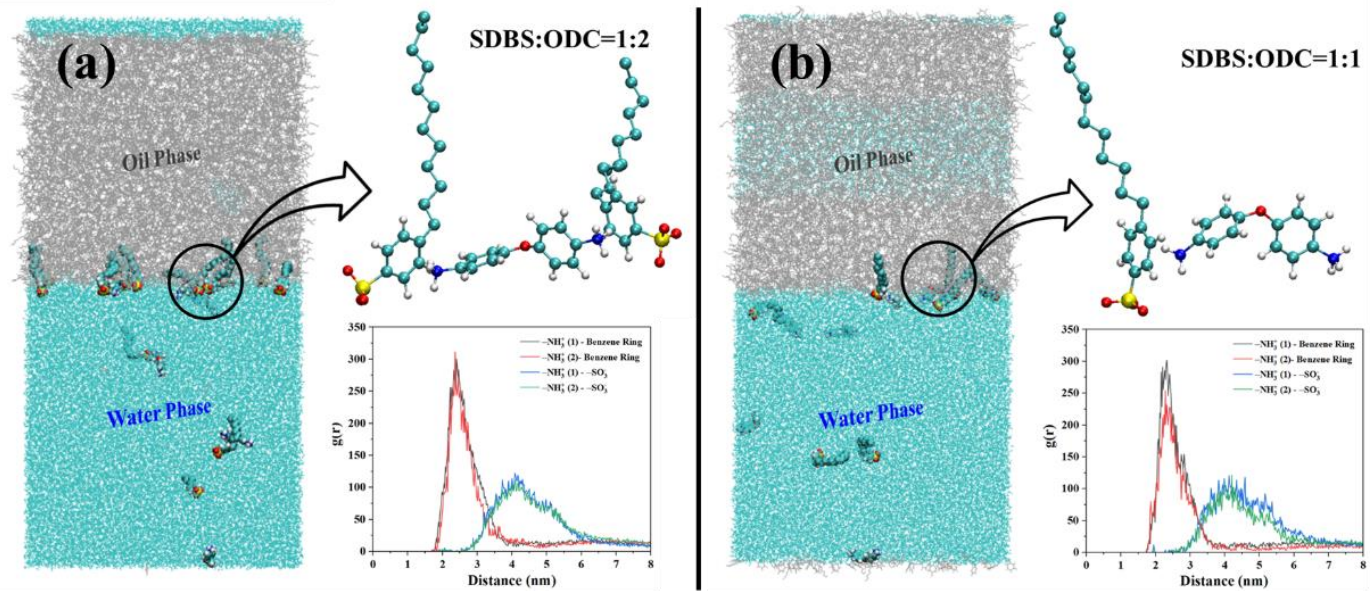

Figure S6. Simulation configurations of the mixed SDBS/ODC system with molecular ratios of 2:1 (a) and 1:1 (b) at the oil/water interface. The oil and water molecules are displayed in silver and blue, respectively. Atom coloring schemes for SDBS and ODC are consistent with those in Fig.4. 


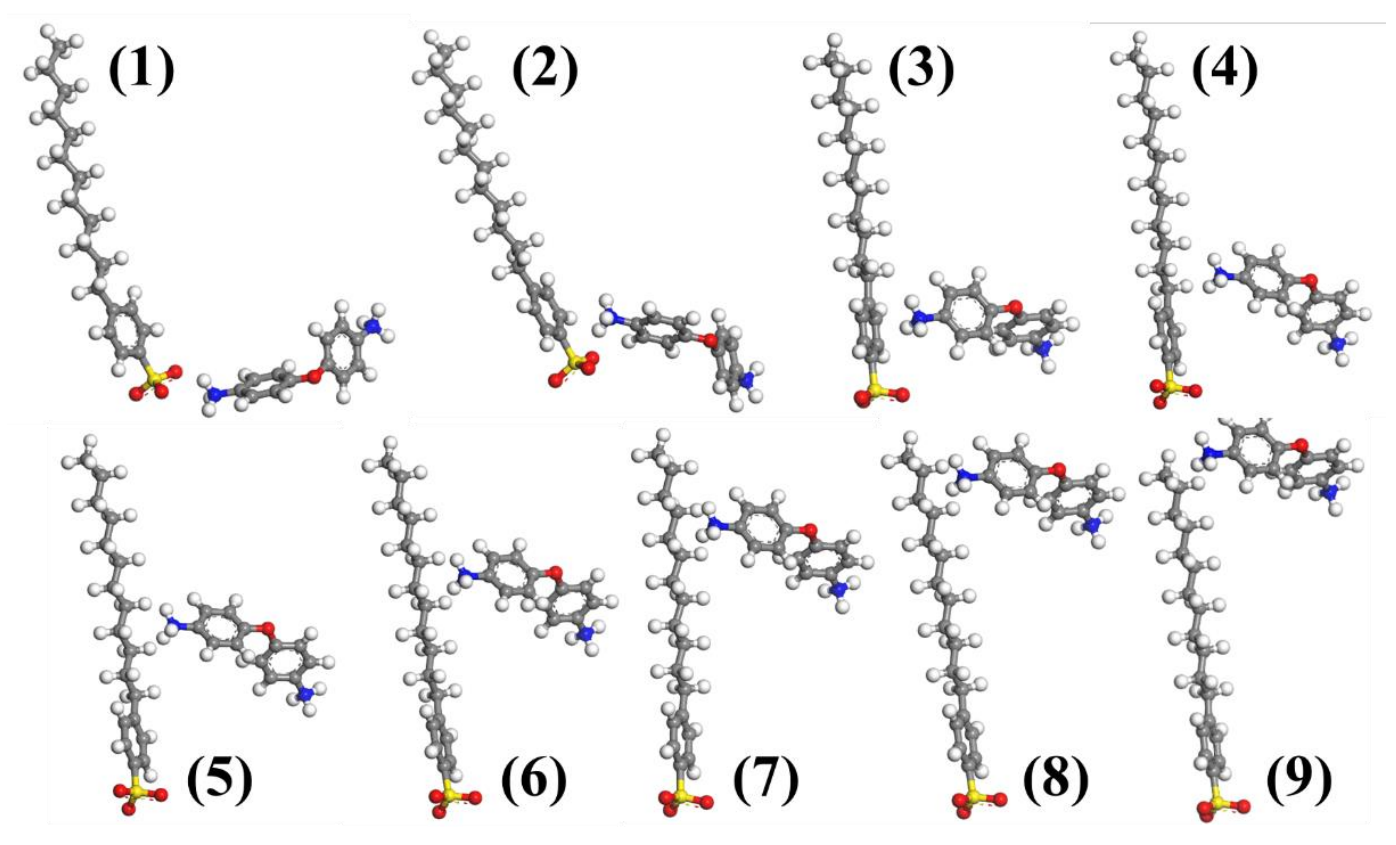

Figure S7. Detailed assembly configurations in the calculation process of SDBS/ODC interaction energy. The configuration order (labels (1) (9)) is consistent with that in Fig.6. 


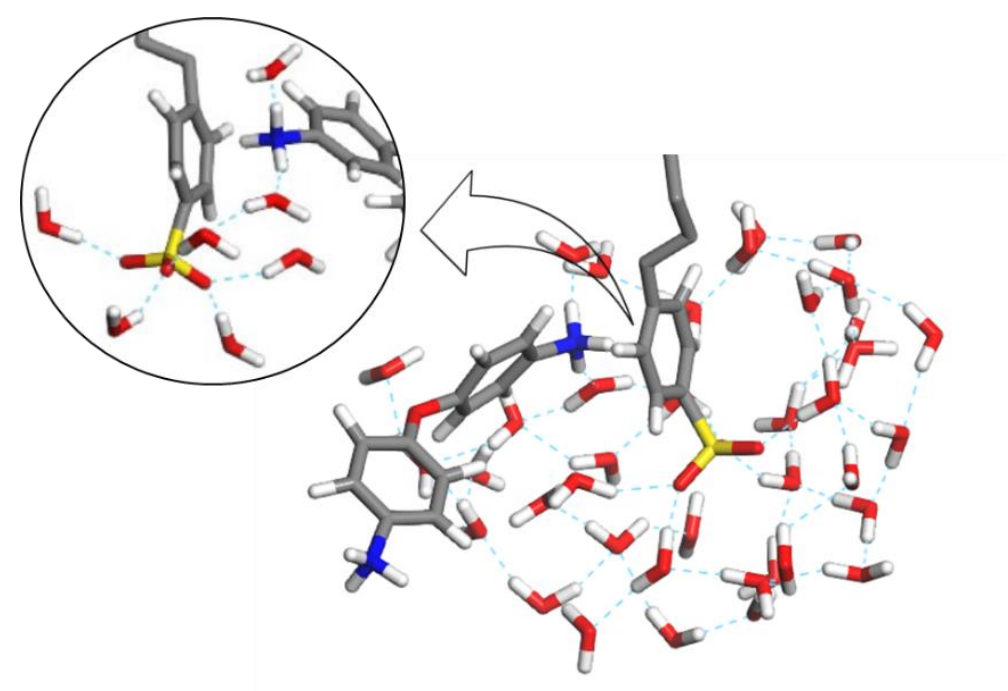

Figure S8. Water molecules around the sulfonate and ammonium groups at the oil/water interface. 